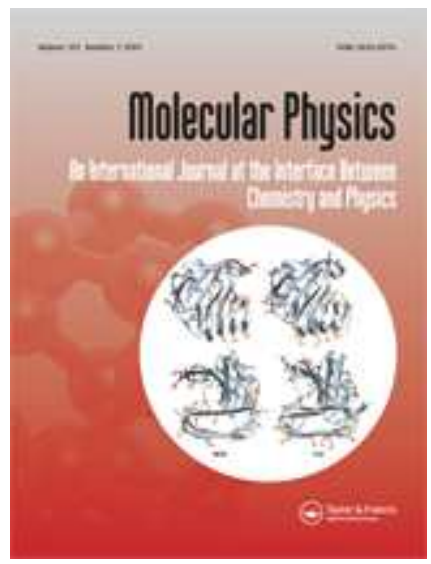

\title{
Tuning the birefringence of the nematic phase in suspensions of colloidal gibbsite platelets
}

\begin{tabular}{|r|l|}
\hline Journal: & Molecular Physics \\
\hline Manuscript ID: & TMPH-2010-0462.R1 \\
\hline Manuscript Type: & Special Issue paper - In honour of Bob Evans \\
\hline Author: & 20-Jan-2011 \\
\hline Complete List of Authors: & $\begin{array}{l}\text { Verhoeff, Aletta; Utrecht University, Van 't Hoff Laboratory for } \\
\text { Physical and Colloid Chemistry } \\
\text { Brand, Rogier; Utrecht University, Van 't Hoff Laboratory for } \\
\text { Physical and Colloid Chemistry } \\
\text { Lekkerkerker, Henk; Utrecht University, Van 't Hoff Laboratory for } \\
\text { Physical and Colloid Chemistry }\end{array}$ \\
\hline Keywords: & liquid crystals, colloids, birefringence, interfaces, magnetic fields \\
\hline \\
\hline $\begin{array}{l}\text { Note: The following files were submitted by the author for peer review, but cannot be converted } \\
\text { to PDF. You must view these files (e.g. movies) online. }\end{array}$ \\
\hline $\begin{array}{l}\text { Verhoeff_Birefringence_resubm.tex } \\
\text { birefringence2.bib }\end{array}$ \\
\hline
\end{tabular}

\section{SCHOLARONE \\ Manuscripts}




\title{
RESEARCH ARTICLE
}

\section{Tuning the birefringence of the nematic phase in suspensions of colloidal gibbsite platelets}

\author{
A. A. Verhoeff*, R. P. Brand and H. N. W. Lekkerkerker \\ Van 't Hoff Laboratory for Physical and Colloid Chemistry, Debye Institute for \\ NanoMaterials Science, Utrecht University, Padualaan 8, 3584 CH, Utrecht, The \\ Netherlands \\ (November 2010)
}

\begin{abstract}
We consider the birefringence patterns of nematic liquid crystals of gibbsite platelets at interfaces and in an aligning magnetic field. In solvents with a refractive index close to the particle refractive index, the intrinsic birefringence of the platelets dominates, resulting in positive birefringence, while if the solvent has a considerably different refractive index, birefringence due to the anisometric particle shape wins over the intrinsic birefringence, resulting in negative birefringence. The inversion of the birefringence leads to fascinating birefringent patterns.
\end{abstract}

Keywords: liquid crystals; colloids; birefringence; interfaces; magnetic fields

\section{Introduction}

Bob Evans has given seminal and lasting theoretical contributions to the statistical physics of liquids, interfacial phenomena and fluids in confining geometries [1-3]. His interest ranges from simple (atomic) fluids, to complex liquids such as colloidal suspensions. He also has a keen interest in experiments, which frequently serve as a source of inspiration for his theoretical work. As a result, his work is equally well appreciated in the theoretical physics community [4] as in the circles of physical chemists [5] and chemical engineers [6].

Less well known is that Bob in his free time (and at his own expense!) is experimenting with a whole series of liquids which in the terminology introduced by Daan Frenkel can be qualified as "nasty" liquids. In the course of these experiments he has recently become interested in the wetting properties of macroscopic plates at glass walls [7]. We felt it therefore fitting to contribute to this volume that is dedicated to him at the occasion of his $65^{t h}$ birthday a paper which deals with a case where wetting of colloidal plates leads to an explanation of birefringence in liquid crystal phases and droplets.

Starting with the work of Einstein [8] and Perrin [9, 10] on the application of statistical mechanics to mesoscopic particles, the analogy between atomic and molecular fluids and colloidal suspensions has been appreciated. A formal basis for this analogy was provided by Onsager [11] and McMillan and Mayer [12]. Apparently,

*Corresponding author. Email: a.a.verhoeff@uu.nl 
the former was the first to coin the phrase "potential of the average force" which for colloidal suspensions replaces the energy in the Maxwell-Boltzmann distribution.

Why study colloidal systems if they simply mimic the behaviour of atoms? One answer is that the interactions between colloidal particles can be tuned from steeply repulsive to deeply attractive and their shapes vary from spherical to highly anisometric rods and plates. This gives rise to unique and fascinating properties ranging from novel phases to remarkable long-lived metastable and non-equilibrium states. These states have been used for detailed studies of fundamental processes of broad relevance such as crystallisation and glass formation $[13,14]$.

Suspensions of highly anisometric particles are known to form a nematic (N) liquid crystalline phase at remarkably low concentration. The formation of the nematic phase was addressed theoretically by Onsager in the 1940s [15], who showed that the I-N phase separation arises from the competition between the orientational entropy and the packing entropy.

Gibbsite $\left(\gamma-\mathrm{Al}(\mathrm{OH})_{3}\right)$ particles represent a unique plate-like system, which displays a nematic phase, both in suspensions of sterically stabilized gibbsite particles in organic solvents [16] as well as in suspensions of charged stabilized gibbsite particles in water [17]. In the former case the interactions are nearly hard, while in the latter case the interactions are soft repulsive and can be tuned by the ionic strength.

Due to the ordering of the particles, the nematic phase displays birefringence. This birefringence is due to form or shape birefringence and in case the particles themselves are optically anisotropic also to intrinsic birefringence [18, 19]. In case of gibbsite these contributions have an opposite sign, and moreover, their relative contribution can be tuned by varying the optical contrast between between the particles and the suspending medium.

The bulk liquid crystal ordering is modified at interfaces (such as the solidfluid and fluid-fluid interface) over length scales that are considerably larger than the particle size, as was shown theoretically [20, 21], experimentally [22] and in simulations [23]. Furthermore, the ordering of liquid crystals can be modified by the aligning action of an externally applied magnetic field [24]. In the case of gibbsite with its tunable birefringence these aligning effects of interfaces and external fields give rise to fascinating phenomena that can be conveniently studied with polarised light microscopy. These effects will be addressed in this paper, which is organized as follows.

In the next section we describe the preparation of the colloidal gibbsite platelets that are subject of investigation. In section 3 we will discuss the theoretical background of intrinsic and form birefringence and show that in the case of gibbsite platelets we have the rather unique situation that depending on the suspending medium, either the one or the other dominates. Experimental details on the polarised light microscopy experiments are given in section 4 . Finally, in section 5 we describe results obtained with gibbsite nematic phase aligned at interfaces and in a magnetic field, as well as confined to droplets, and discuss what these experiments teach us about the optical properties of gibbsite liquid crystals.

\section{Preparation of colloidal gibbsite}

Colloidal gibbsite $\left(\gamma-\mathrm{Al}(\mathrm{OH})_{3}\right)$ platelets were synthesized by hydrothermal treatment of aluminum alkoxides in acidic environment, a procedure developed in our laboratory [25]. To that end, an acidic aqueous solution containing hydrochloric acid ( $\mathrm{HCl} 0.09$ M, 37\%, Merck), aluminium sec-butoxide (0.08 M, 95\%, Fluka Chemika) and aluminium iso-propoxide (0.08 M, 98+\%, Acros Organics) was pre- 
pared and mechanically stirred for 10 days. Subsequently, the mixture was heated in a glass reaction vessel in a water bath at $85{ }^{\circ} \mathrm{C}$ for $72 \mathrm{~h}$. Then, the colloidal dispersion was centrifuged at $1000 \mathrm{~g}$ (overnight, 15-20 h) in order to remove the smallest particles and decrease the polydispersity. The resulting dispersion was dialyzed against demineralized water in tubes of regenerated cellulose (Visking, MWCO 12 000-14 000) until the conductivity dropped to $20 \mu \mathrm{S} \mathrm{cm}^{-1}$.

The obtained particles were characterised with transmission electron microscopy (Tecnai 10, FEI Company), see Figure 1(a). The hexagonal platelets typically have a diameter of about $200 \mathrm{~nm}$ and a thickness of $8 \mathrm{~nm}$.

Subsequently, the obtained particles were treated either to enhance the stability in aqueous dispersions, or to enable dispersion in apolar solvents.

For enhanced stability in aqueous dispersions, $0.5 \mathrm{w} / \mathrm{w} \%$ aluminum chlorohydrate (Locron P, Hoechst AG, Germany) was added to the dispersion. The enhanced stability is associated with the presence of aluminum polycations $\left(\mathrm{Al}_{13}\right.$ Keggin ions, $\left.\left[\mathrm{Al}_{13} \mathrm{O}_{4}(\mathrm{OH})_{24}\left(\mathrm{H}_{2} \mathrm{O}\right)_{12}\right]^{7+}\right)$ that form at $\mathrm{pH} \approx 4[26]$ and adsorb on the surface of the gibbsite particles. This gives rise to a steep repulsive particle interaction which prevents the platelets from aggregating [27]. After three days, the dispersion was centrifuged to remove excess aluminum chlorohydrate and the particles were subsequently redispersed in a $10^{-2} \mathrm{M} \mathrm{NaCl}$ solution.

To enable dispersion of the gibbsite platelets in apolar solvents the particles were sterically stabilized with a amino-modified poly-iso-butylene (SAP 230 TP, Infineum, UK), by following two different procedures.

The first procedure involves a gradual substitution of the solvent upon addition of the stabilizing polymer, as described by [16]. The particles obtained through this method were dispersed in 2-bromotoluene (Acros, 99\%).

Following an alternative method, the gibbsite platelets were freeze-dried in the presence of poly-iso-butylene in 1-propanol (Acros, 99\%) [28, 29]. The thus stabilized particles were dispersed in toluene (J.T. Baker, 99.5\%).

Finally, the dispersions were concentrated with centrifugation and subsequent redispersion to reach the desired particle concentration. 


\section{Birefringence}

At sufficiently high concentration suspensions of gibbsite particles form nematic liquid crystal phases in which the platelets possess orientational ordering. As a result, the nematic phase is birefringent, and as is shown in Figure 1(b) light is transmitted when it is placed between crossed polarisers. The isotropic phase is dark, but can be made birefringent by gently moving the sample so that the platelets align in the resulting flow.

Birefringent materials have two or more refractive indices. Here, we will focus on uniaxial materials, with two refractive indices. If linearly polarised light enters such a material, it is refracted into two components with orthogonal vibration directions, the ordinary ray (o-ray) with the polarisation perpendicular and the extraordinary ray (e-ray) parallel to the axis of anisotropy. The birefringence is defined as $\Delta n \equiv n_{e}-n_{o}$, with the $n_{e}$ and $n_{o}$ the refractive indices for the e-ray and the o-ray. Because of the different refractive indices for these rays, they travel with a different velocity through the material, giving rise to a phase difference $\delta$ :

$$
\delta=\frac{2 \pi}{\lambda}\left(n_{e}-n_{o}\right) t
$$

with $\lambda$ the wavelength of the incident light and $t$ the path length through the material [30]. The value $\left|\left(n_{e}-n_{o}\right)\right| t$ is referred to as retardation or optical path difference (OPD) [31]. When the refracted light arrives at the second polariser (also, analyser) that is at a $90^{\circ}$ angle with respect to the first one, only the component with the vibration direction parallel to the polariser will pass through. The resulting intensity is given by:

$$
I=E^{2} \sin ^{2} 2 \theta \sin ^{2} \frac{\delta}{2}
$$

with $E$ the amplitude of the electro-magnetic vector and $\theta$ the angle between the polarisation of the incident light and the direction in the plane perpendicular to the direction of propagation in which the refractive index corresonds to $n_{o}$ [30]. We will find a minimum intensity behind the analyser if $\theta=0, \pi / 2, \pi, \ldots$ and relative maxima for $\theta=\pi / 4,3 \pi / 4,5 \pi / 4, \ldots$ with values of:

$$
I_{\max }=E^{2} \sin ^{2} \frac{\delta}{2} .
$$

Due to the wavelength dependence of $\delta$, the use of white light leads to beautiful interference (or retardation) colours, as part of the wavelengths will interfere constructively and others destructively. These colours occur in a specific sequence, known as Newton's colour scale and are often presented as a function of retardation in the form of a Michel-Levy chart. Figure 2 shows the colour sequence visualised with a Babinet compensator, a tunable retardation plate that is composed of two opposed quartz wedges [32].

One can distinguish two types of birefringence: intrinsic and form birefringence. Intrinsic birefringence is caused by the anisotropy of the crystal lattice and therefore a fixed material parameter.

Gibbsite $\left(\gamma-\mathrm{Al}(\mathrm{OH})_{3}\right)$ has a monoclinic crystal lattice with the following refractive indices: natural gibbsite $\alpha \simeq 1.56-1.58, \beta \simeq 1.56-1.58$, and $\gamma \simeq 1.58-1.60$. For synthetically prepared gibbsite $\alpha=\beta=1.577$ and $\gamma=1.595$ has been reported [33]. The optic axial plane (where the refractive index does not depend on the polarization direction of the incident light) is perpendicular to the (010) plane, and 


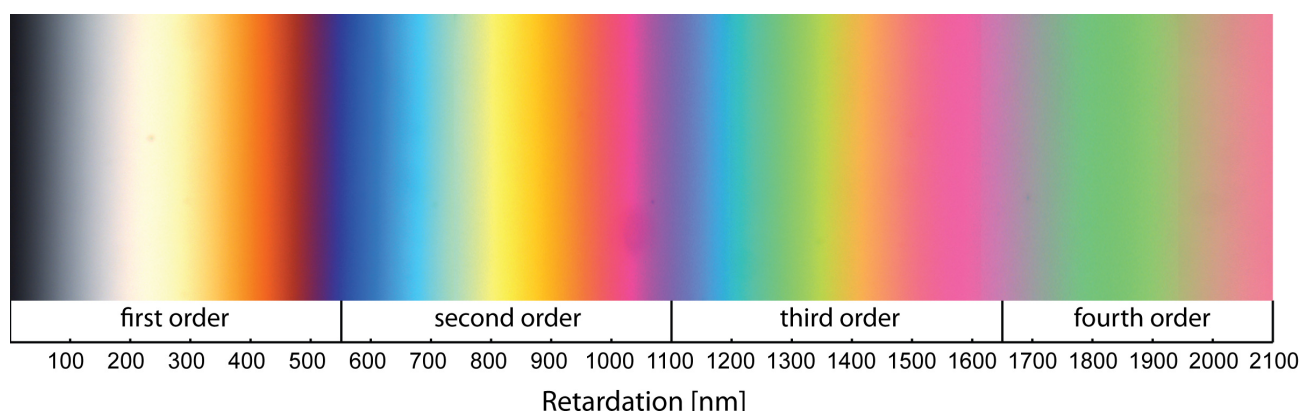

Figure 2. Newton's colour scale, here visualised with a Babinet compensator, represents the retardation colour sequence as a function of retardation.
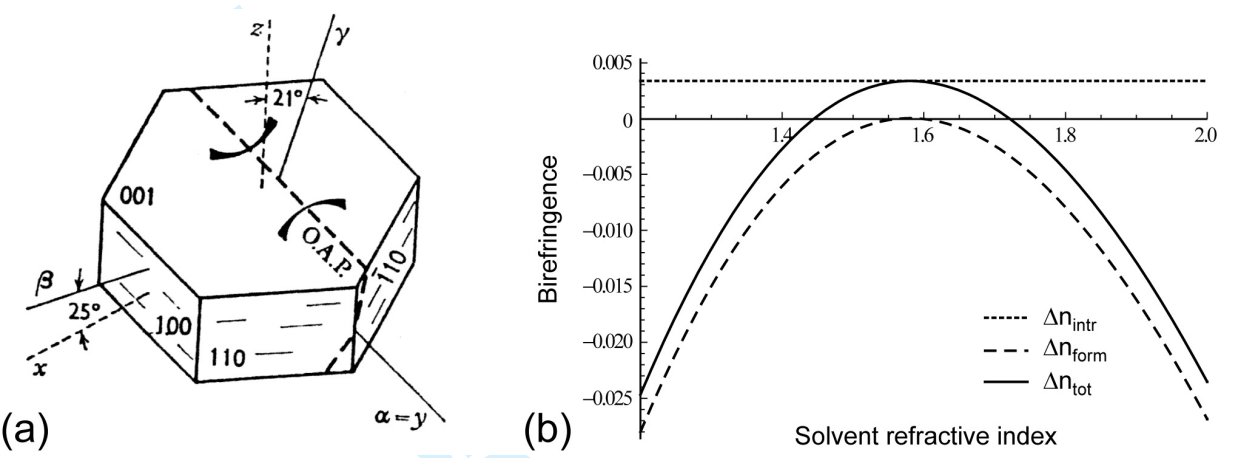

Figure 3. (a) Crystallographic and optical axes of gibbsite. Reprinted from [33] with permission of The Geological Society London. (b) Intrinsic, form and total birefringence of a nematic phase of colloidal gibbsite as a function of solvent refractive index for $\phi=0.26, S_{2}=0.8, L_{a}=0.03$ and $L_{b}=0.94$.

the extinction angle makes a angle of $-21^{\circ}$ with the z-axis [33].

For colloidal gibbsite, which consists of hexagonal platelets, this implies that the largest refractive index, or slow axis, has an angle of $-21^{\circ}$ with respect to the particle director (see Figure 1). However, in the nematic phase, although the platelets have an net average orientation of the particle director, in the particle plane all orientations are allowed. The resulting intrinsic birefringence of a platelet is therefore effectively parallel to the director, with $\Delta n_{i} \approx(\gamma-\alpha) \cos \left[21^{\circ}\right]=0.016$. Following $[34,35]$ the birefringence of a suspension of platelets due to the intrinsic particle birefringence is given by

$$
\Delta n_{\text {intr }}=\Delta n_{i} \phi S_{2}
$$

with $\phi$ the particle volume fraction and $S_{2}$ the nematic order parameter.

On the other hand, form (or shape) birefringence arises when anisotropic molecules or particles are dispersed in an ordered arrangement in a liquid with a different refractive index. It depends on the particle shape and the solvent refractive index and can therefore be manipulated.

Wiener [18] showed that an assembly of thin parallel plates in a medium with a different refractive index behaves like a negative uniaxial crystal. Bragg and Pippard [19] extended his work to ellipsoids and obtained for the form birefringence of an assembly of particles with refractive index $n_{p}$ in a solvent with $n_{s}$ :

$$
\Delta n_{f o r m}=n_{a}-n_{b}=\phi\left(n_{p}-n_{s}\right) \frac{9 k}{(3+k)^{2}}\left(L_{b}-L_{a}\right)
$$

with $\phi$ the particle volume fraction, $k=(1-\phi)\left(n_{p}^{2} / n_{s}^{2}-1\right)$, and $L_{a}$ and $L_{b}$ de- 
polarizing factors that depend on the particle symmetry. To obtain a reasonable estimate for the form birefringence of gibbsite where the particles have intrinsic birefringence as well, we can use this equation with an average of the three refractive indices, $n_{p}=1.58$. Although this is not exact, according to Twersky [36] it should introduce little error because the refractive indices are close. In their derivation Bragg and Pippard considered an assembly with perfectly aligned particles (orientational order parameter $S_{2}=1$ ), which is obviously not true for the gibbsite nematic phase. To account for this, we multiply by $S_{2}[34,35]$, analogous to Equation 4. Previous experiments on the alignment of the gibbsite nematic phase in a magnetic field established typically $S_{2} \approx 0.8$ [37].

In Figure 3(b) we plot the intrinsic and form birefringence as a function of solvent refractive index $n_{s}$, for a volume fraction $\phi$ of 0.26 . The depolarizing factors can be calculated by considering the particles as ellipsoids [38], resulting in $L_{a}=0.03$ and $L_{b}=0.94$ for a bare platelet with an aspect ratio of $25 . \Delta n_{t o t}$ is obtained by simply taking the sum of the two contributions. We find that the birefringence of a gibbsite nematic phase is negative for most solvents, except if the refractive index is close to that of gibbsite itself.

\section{Polarised light microscopy}

Birefringence of the gibbsite nematic phase was studied with polarised light microscopy, using a Nikon LV100Pol microscope, equipped with a $2 \times$ Nikon CFI Plan UW objective and a $10 \times$ Nikon CFI Plan Fluor ELWD objective. The microscope was in a tilted position with the focal plane along gravity in order to allow investigation of the samples in vertical position. Images were captured with a MicroPublisher 5 megapixel ccd camera (MP5, QImaging).

To determine the sign of the birefringence a full wave $(\lambda=530 \mathrm{~nm})$ retardation plate was used, which is a birefringent filter with a known birefringence and orientation. It is placed in the light path between the polariser and analyser at an angle of $45^{\circ}$ for maximum effect, and it adds a fixed retardation to all the wave fronts in the optical path. Depending on the respective orientation of the slow axes of the liquid crystal and the retardation plate, the retardation is added (parallel orientation) or subtracted (perpendicular orientation).

To align the nematic phase in a magnetic field a home-built polarization microscope setup was used, assembled from the head of a Zeiss Axiolab microscope, equipped with crossed polarisers, and a $2 \times$ Edmund Optics objective. The magnetic field was generated with a Bruker BE25v Electromagnet, equipped with large flat pole shoes producing a very homogeneous magnetic field in the sample volume. Field strengths ranged from $B=0-1.8 \mathrm{~T}$ and were monitored with a LakeShore 421 Gaussmeter. The samples could be rotated along their vertical axis with adjustable velocity (usually $\sim 0.25 \mathrm{~Hz}$ ).

Samples were prepared in flat optical capillaries (VitroCom, NJ, USA), with internal dimensions of $0.1 \times 2 \times 40 \mathrm{~mm}^{3}$ for the experiments at the isotropic-nematic interface and in magnetic field and $0.2 \times 4 \times 40 \mathrm{~mm}^{3}$ for study of tactoids, and flame-sealed to avoid evaporation of the solvent. The samples were homogenized and left to phase separate, which evolved via the formation, sedimentation and subsequent coalescence of tactoids. 


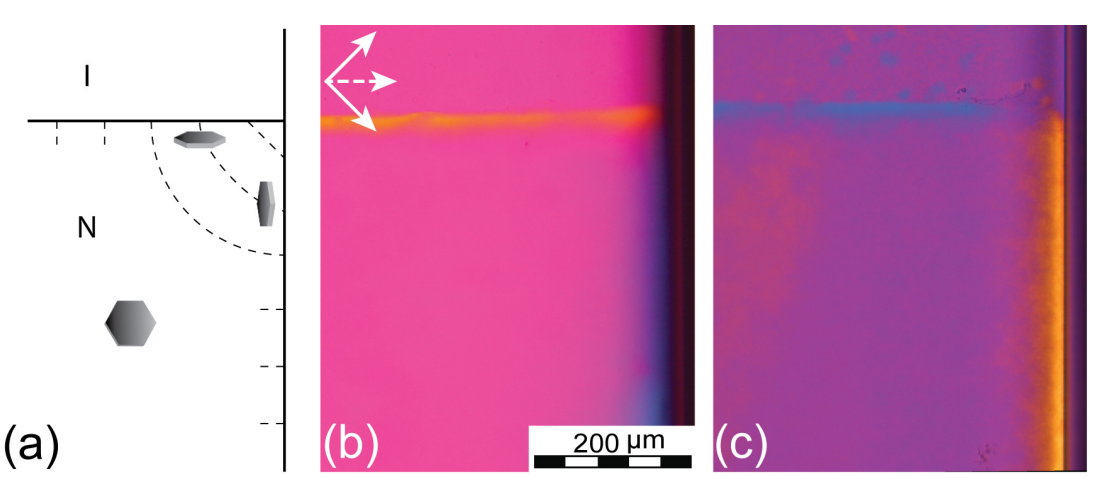

Figure 4. Alignment of the nematic phase at the isotropic-nematic interface and the capillary walls; schematically depicted in (a) and observed with polarised light microscopy in a system of gibbsite dispersed in toluene (b) and water (c).

\section{Results}

\subsection{Anchoring at a planar wall and the isotropic-nematic interface}

The isotropic-nematic phase separated sample shown in Figure 1(b), was imaged just after phase separation. The nematic phase displays bright colourful birefringence, indicating the presence of many domains with different orientations. Over a period of days to weeks (depending, among other things, on the sample thickness) these domains coalesce and align in such a way that most platelets have the preferred anchoring with the interface. For entropic reasons, colloidal platelets exhibit homeotropic anchoring, which means that they tend to align with the director perpendicular to the isotropic-nematic interface as well as to hard walls [22, 39], as illustrated in Figure 4(a).

We will now use this preferential alignment to study the birefringence of the gibbsite nematic phase in toluene with refractive index $n_{s}=1.4969$ and compare that to the gibbsite nematic phase in water with $n_{s}=1.333$.

Figure 4(b) and (c) show two gibbsite systems, both one week after phase separation. The sample in Figure 4(b) is a suspension in toluene with an overall volume fraction $\phi=0.22$, while the sample in Figure 4(c) is a dispersion of gibbsite in an aqueous salt solution $\left(10^{-2} \mathrm{M} \mathrm{NaCl}\right)$ at $\phi=0.25$.

The appearance of the nematic phase is clearly different from the freshly phase separated sample in Figure 1(b). The bulk nematic phase now appears with the same colour as the isotropic phase, the typical magenta colour of the retardation plate independent of the orientation of the polarisers, which implies that it does not exhibit birefringence. Thus the platelets are oriented with their director pointing into the focal plane, which indeed corresponds to homeotropic alignment with the capillary walls. On the other hand, along the isotropic-nematic interface and along the capillary wall at the side we observe birefringent bands with different colours. This indicates that the nematic director points in the vertical and horizontal direction, corresponding to the expected alignment shown in Figure 4(a).

In the suspension with sterically stabilized gibbsite in toluene (Figure 4(b)) the nematic phase near the isotropic-nematic interface appears orange, which corresponds to a subtraction of the retardation with respect to the retardation plate (see Figure 2). On the other hand, a blue retardation colour can be observed near the capillary wall, corresponding to an addition of the optical path difference. From these observations we can deduce that the slow axis is parallel to the platelet director and hence that intrinsic birefringence is dominant.

If we now compare this to the aqueous suspension shown in Figure 4(c), we observe that the retardation colours are reversed: blue at the isotropic-nematic 


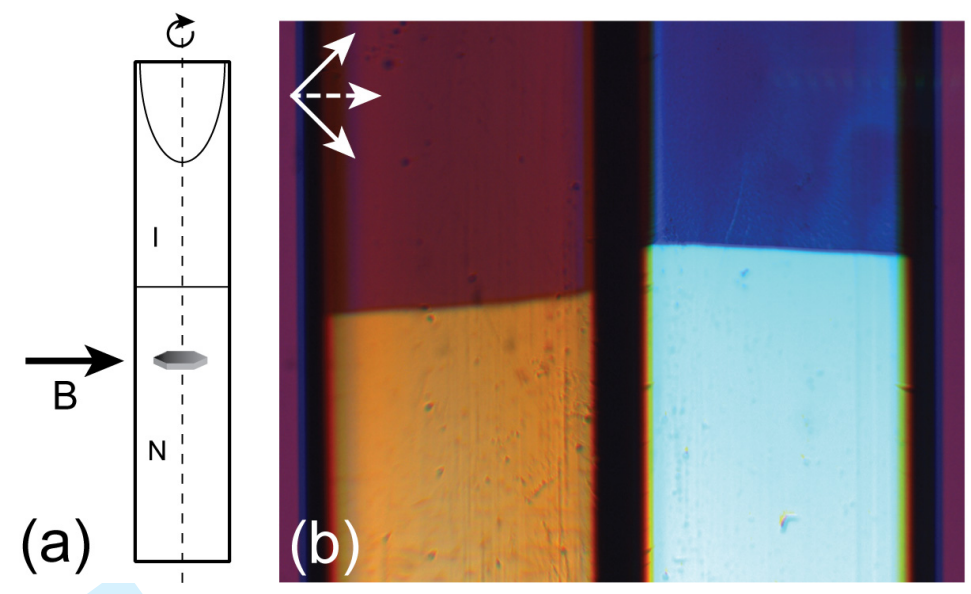

Figure 5. (a) Rotation of the sample around its vertical axis in a magnetic field aligns the platelets with the director in the vertical direction. (b) Isotropic-nematic phase separated suspension of sterically stabilized gibbsite (left) and charge stabilized gibbsite (right) after one hour rotation in a magnetic field with a strength of $1.8 \mathrm{~T}$. Solid arrows indicate the polarisers, dashed arrow the orientation of the retardation plate. Scale: the capillaries are $2 \mathrm{~mm}$ wide.

interface and orange near the capillary wall. This implies that the slow axis is now perpendicular to the platelet director and thus that in water, with a much larger difference between the refractive index of particle and solvent, form birefringence wins over the intrinsic birefringence.

\subsection{Nematic phase aligned in a magnetic field}

One could argue that the difference in particle stabilisation between the two systems could influence the anchoring preference. For instance, in the case of thermotropic liquid crystals it is well-known that chemical interaction rather than molecular shape determines the preferred anchoring [40]. A convenient way to exclude the influence of this effect, is to align the nematic phase of both samples in a magnetic field.

Colloidal gibbsite platelets have an anisotropic magnetic susceptibility, which implies that an external magnetic field gives rise to an induced magnetic moment in the particles. Previous studies on sterically stabilized gibbsite suspensions [22, 24] established that the magnetic anisotropy of these platelets is negative $\left(\Delta \chi=-10^{-22}\right.$ $\left.\mathrm{J} / \mathrm{T}^{2}\right)$, and therefore the platelets tend to align with their director perpendicular to an externally applied magnetic field. This implies that the director orientation is restricted to the plane perpendicular to the magnetic field direction. If instead of a static field a rotating field is used, the platelets will align in a single direction, with the director perpendicular to the rotation plane. Rotation of the sample in a static field (like sketched in Figure 5(a)) has the same effect, and it has been shown that one can obtain a single domain nematic phase with a well-defined orientation $[24]$.

Figure 5(b) shows the two isotropic-nematic phase separated suspensions after rotation at $0.25 \mathrm{~Hz}$ for one hour in a magnetic field with a field strength of 1.8 T. There is a distinct difference in the retardation colours of the samples, in the isotropic phase and even more clearly in the nematic phase. With the retardation plate in the horizontal direction the retardation colour of the sample in toluene corresponds to first order orange, implying a subtraction of the optical path difference with roughly $140 \mathrm{~nm}$ (see Figure 2). On the other hand, the aqueous sample displays a second order blue tint, corresponding to an addition of the optical path difference with about $210 \mathrm{~nm}$. 
With a capillary inner thickness of $100 \mu \mathrm{m}$ this corresponds to a $\Delta n$ for toluene of $1.4 \cdot 10^{-3}$ and $\Delta n=-2.1 \cdot 10^{-3}$ for aqueous gibbsite. By combining Equation 4 and 5 in section 3 , we find $\Delta n=2 \cdot 10^{-3}$ for gibbsite dispersed in toluene, in line with the experimentally obtained value. For aqueous gibbsite we obtain $\Delta n=-8 \cdot 10^{-3}$, which is somewhat lower than the experimental value, but still in reasonable agreement taking into account the approximations with regard to the order parameter and the volume fraction of the nematic phase.

The fact that the isotropic phases of the two samples also display a colour difference, both deviating from the typical magenta colour of the retardation plate, indicates that the particles in the isotropic phase also align in the magnetic field, in that way forming a paranematic phase. The paranematic phase in toluene displays an orange-red tint, corresponding to a subtraction of the retardation, whereas the paranematic phase in the aqueous sample is purple-blue, corresponding to an addition of the retardation.

From these results we find again that for gibbsite dispersed in toluene the birefringence is positive, corresponding to the intrinsic birefringence of the platelets. On the other hand, for aqueous gibbsite, with a much larger difference between the refractive index of particle and solvent, the birefringence is negative, implying that form birefringence is dominant over the intrinsic particle birefringence.

This is in line with what we obtained from the anchoring at the wall and the isotropic-nematic interface, implying that particle stabilisation does not affect the anchoring preference and is indeed a result of the particle shape. However, the absolute magnitude of the anchoring strength does depend on particle stabilisation and solvent, as we have shown in previous publications [41, 42].

\section{3. $\quad$ Tactoids with radial director field}

An alternative method to study the birefringence of the nematic phase is to investigate the nematic phase confined to micrometer-sized droplets. The properties of such droplets, or tactoids, are determined by a competition between surface anchoring and bulk elastic free energy [41]. The balance of these two depends on the droplet size, which in the case of plate-like particles leads to oblate shaped droplets with a uniform director field for tactoids smaller than the ratio of the splay elastic constant and the anchoring strength $K_{1} / w$. However, tactoids larger than $K_{1} / w$ are spherical with a radial director field and a point defect in the center, a so-called hedgehog, as depicted schematically in Figure 6(a). Here, we focus on the latter type of tactoid.

Figures 6(b) and (c) display two radial tactoids, one in a suspension in bromotoluene and the other in an aqueous gibbsite suspension. The refractive index of bromotoluene is very close to that of gibbsite $\left(n_{s}=1.556\right.$ and $n_{s} \approx 1.58$ respectively), so that the gibbsite platelets are almost index matched. We observe the typical birefringence pattern of a radial tactoid, consisting of four parts with the opposite quarters the same retardation colour. The retardation colours of the tactoids in the two solvents are reversed: the quarters that are blue in the one are yellow/orange in the other and vice versa. This confirms again the inversion of the birefringence observed above, with in bromotoluene the slow axis along and in water perpendicular to the nematic director. 


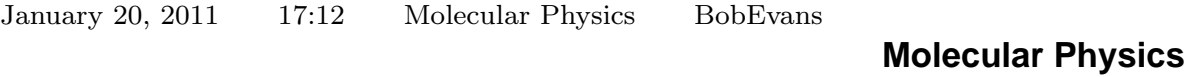

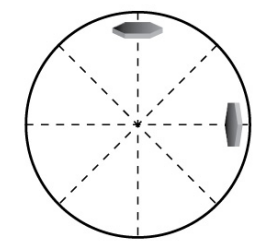

(a)
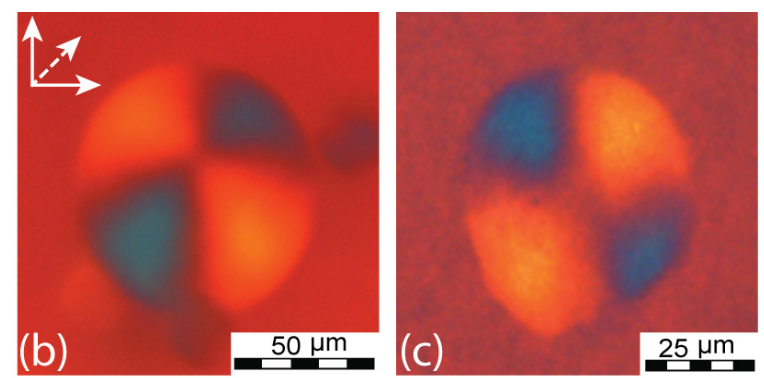

Figure 6. Tactoid with radial director field and hedgehog defect; schematically depicted in (a) and observed with polarised light microscopy in a gibbsite suspension in bromotoluene (b) and in water (c).

\section{Conclusions}

We have demonstrated that it is possible to tune the birefringence of the nematic phase in suspensions of gibbsite platelets by varying the solvent refractive index. In solvents with a refractive index close to that of the gibbsite platelets, such as toluene and bromotoluene, the positive intrinsic birefringence of the particles determines the birefringence of the nematic phase, while in solvents with a considerably lower refractive index, such as water, form birefringence wins over the intrinsic birefringence, resulting in a net negative birefringence of the nematic phase. In conjunction with the anchoring properties of interfaces and the aligning influence of an external magnetic field this leads to fascinating birefringence patterns.

\section{Acknowledgement}

A.A.V. would like to thank the Royal Netherlands Academy of Arts and Sciences (KNAW) for financial support.

\section{References}

[1]R. Evans, The nature of the liquid-vapour interface and other topics in the statistical mechanics of non-uniform, classical fluidsAdv. Phys. 28, 143-200 (1979).

[2]P. Tarazona and R. Evans, A simple density functional theory for inhomogeneous liquids. Wetting by gas at a solid-liquid interfaceMol. Phys. 52, 847-857 (1984).

[3]P. Tarazona, U.M.B. Marconi and R. Evans, Phase equilibria of fluid interfaces and confined fluids. Non-local versus local density functionalsMol. Phys. 60, 573-595 (1987).

[4]R. Evans and J.R. Henderson, Pair correlation function decay in models of simple fluids that contain dispersion interactionsJ. Phys.: Condens. Matter 21, 474220 (2009).

[5]M. Schmidt, H. Löwen, J.M. Brader and R. Evans, Density functional for a model colloid-polymer mixturePhys. Rev. Lett. 85, 1934-1937 (2000)

[6]R. Evans, Fluids adsorbed in narrow pores: phase equilibria and structureJ. Phys.: Cond. Matter 2, 8989-9007 (1990).

[7]Personal homepage Bob Evans . <http://www.phy.bris.ac.uk/people/evans_r/experiments.html>.

[8]A. Einstein, On the motion, required by the molecular-kinetic theory of heat, of particles suspended in a fluid at restAnn. Phys. 17, 549-560 (1905).

[9]J. Perrin, Mouvement brownien et réalité moléculaireAnn. Chim. Phys. 18, 5-114 (1909).

[10] J. Perrin, Les atomes (Alcan, Paris, 1913).

11]L. Onsager, Theories of Concentrated ElectrolytesChem. Rev. 13, 73-89 (1933).

[12] W.G. McMillan and J.E. Mayer, The statistical thermodynamics of multicomponent systemsJ. Chem. Phys. 13, 276-305 (1945).

[13] P.N. Pusey and W. van Megen, Phase behaviour of concentrated suspensions of nearly hard spheresNature 320, 340-342 (1986).

[14]P.N. Pusey, Colloidal glassesJournal of Physics-Condensed Matter 20 (49), 494202 (2008).

[15]L. Onsager, The effect of shape on the interaction of colloidal particlesAnn. NY Acad. Sci. 51, 627-659 (1949).

[16]F.M. van der Kooij and H.N.W. Lekkerkerker, Formation of nematic liquid crystals in suspensions of hard colloidal plateletsJ. Phys. Chem. B 102 (40), 7829-7832 (1998).

[17]D. van der Beek and H.N.W. Lekkerkerker, Nematic ordering vs. gelation in suspensions of charged plateletsEurophys. Lett. 61, 702-707 (2003). 
[18] O. Wiener, Abh. Sächs. Ges. Akad. Wiss., Math.-Phys. Kl. 32, 575 (1912).

[19]W.L. Bragg and A.B. Pippard, The Form Birefringence of MacromoleculesActa Cryst. 6, 865-867 (1953).

[20]L. Harnau and S. Dietrich, Fluids of platelike particles near a hard wallPhys. Rev. E 65, 021505 (2002).

[21]M. Bier, L. Harnau and S. Dietrich, Free isotropic-nematic interfaces in fluids of charged platelike colloidsJ. Chem. Phys. 123, 114906 (2005).

[22]D. van der Beek, H. Reich, P. van der Schoot, M. Dijkstra, T. Schilling, R. Vink, M. Schmidt, R. van Roij and H. Lekkerkerker, Isotropic-nematic interface and wetting in suspensions of colloidal plateletsPhys. Rev. Lett. 97 (8), 087801 (2006).

[23] M.M. Pineiro, A. Galindo and A.O. Parry, Surface ordering and capillary phenomena of confined hard cut-sphere particlesSoft Matter 3, 768-778 (2007).

[24]D. van der Beek, P. Davidson, H.H. Wensink, G.J. Vroege and H.N.W. Lekkerkerker, Influence of a magnetic field on the nematic phase of hard colloidal plateletsPhys. Rev. E 77 (3), 031708 (2008).

[25] A.M. Wierenga, T.A.J. Lenstra and A.P. Philipse, Aqueous dispersions of colloidal gibbsite platelets: synthesis, characterisation and intrinsic viscosity measurementsColloids Surf. A 134, 359-371 (1998).

[26]J. Hernandez, Thèse de Doctorat de l'Université Pierre et Marie Curie, Ph.D. thesis, Université Pierre et Marie Curie 1998.

[27]M.P.B. van Bruggen, M. Donker, H.N.W. Lekkerkerker and T.L. Hughes, Anomalous stability of aqueous boehmite dispersions induced by hydrolyzed aluminium poly-cationsColloids Surf. A 150, 115-128 (1999).

[28]L. Heux, G. Chauve and C. Bonini, Nonflocculating and Chiral-Nematic Self-ordering of Cellulose Microcrystals Suspensions in Nonpolar SolventsLangmuir 16 (21), 8210-8212 (2000).

[29] M.C.D. Mourad, E.J. Devid, M.M. van Schooneveld, C. Vonk and H.N.W. Lekkerkerker, Formation of Nematic Liquid Crystals of Sterically Stabilized Layered Double Hydroxide PlateletsJ. Phys. Chem. B 112 (33), 10142-10152 (2008).

[30]M. Born and E. Wolf, Principles of Optics, Electromagnetic Theory of Propagation, Interference and Diffraction of Light $(,, 1998)$.

[31]P.C. Robinson and S. Bradbury, Qualitative Polarized-Light Microscopy (, , 1992).

[32] N.H. Hartshorne, The microscopy of liquid crystals Microscope series (Microscope Publications Ltd., London, 1974).

[33]J.F. Bowles, D.J. Vaughan, R.A. Howie and J. Zussman, Rock-Forming Minerals Volume 5A: NonSilicates: Oxides, Hydroxides and Sulphides (2nd Edition) (, , 2011).

[34]S. Neveu-Prin, F.A. Tourinho, J.C. Bacri and R. Perzynski, Magnetic birefringence of cobalt ferrite ferrofluidsColloids Surf. A 80, 1-10 (1993).

[35]B.J. Lemaire, P. Davidson, J. Ferré, J.P. Jamet, D. Petermann, P. Panine, I. Dozov and J.P. Jolivet, Physical properties of aqueous suspensions of goethite nanorodsEur. Phys. J. E 13, 291-308 (2004).

[36]V. Twersky, Form and intrinsic birefringenceJ. Opt. Soc. Am. 65 (3), 239-245 (1975).

[37]D. van der Beek, A.V. Petukhov, P. Davidson, J. Ferr é, J.P. Jamet, H.H. Wensink, G.J. Vroege, W Bras and H.N.W. Lekkerkerker, Magnetic-field-induced orientational order in the isotropic phase of hard colloidal plateletsPhys. Rev. E 73 (4), 041402 (2006).

[38] J.A. Osborn, Demagnetizing Factors of the General EllipsoidPhys. Rev. 67, 351-357 (1945).

[39]R.H.J. Otten and P. van der Schoot, Capillary rise of an isotropic-nematic fluid interface: surface tension and anchoring versus elasticityLangmuir 25, 2427-2436 (2009).

[40]P.G. de Gennes, The Physics of Liquid Crystals (, , 1974).

[41]A.A. Verhoeff, R.H.J. Otten, P. van der Schoot and H.N.W. Lekkerkerker, Magnetic field effects on tactoids of plate-like colloidsJ. Chem. Phys. 134 (2011).

[42] A.A. Verhoeff, I.A. Bakelaar, R.H.J. Otten, P. van der Schoot and H.N.W. Lekkerkerker, Tactoids of plate-like particles: size, shape and director fieldLangmuir 27 (1), 116-125 (2010). 